\title{
ANAESTHETIC CHALLENGES FOR A RARE CASE OF \\ A PARTURIENT WITH PARAMYOTONIA CONGENITA AND TERMINAL FILUM LIPOMA PRESENTING FOR LABOUR AND CAESAREAN SECTION UNDER EPIDURAL ANAESTHESIA
}

\author{
W. Siow 1 , A. Chiew ${ }^{2}$. \\ 1SingHealth Anaesthesiology Residency Programme, Anaesthesiology, Singapore \\ 2National Healthcare Group Anaesthesiology Residency Programme, Anaesthesiology, Singapore
}

\begin{abstract}
We describe a parturient with paramyotonia congenita (PMC) and terminal filum lipoma who presented for induction of labour followed by emergency caesarean section under epidural anaesthesia. She subsequently developed postpartum paraparesis which self-resolved.

\section{INTRODUCTION}

Paramyotonia Congenita

- A form of periodic paralysis characterized by irregular episodes of flaccid paresis and stiffness exacerbated during exercise or cold

- Rare autosomal dominant condition

- Affect fewer than 1 in 100,000

- Due to mutation in SCN4A gene encoding skeletal muscle sodium channels, therefore causing dysregulation of sodium ion flows into muscle cells resulting in abnormal contraction and relaxation [1,2]

\section{Terminal Filum Lipoma}

- Most common intra-spinal lipomas

- Usually incidental findings and asymptomatic

- Rarely may result in tethered spinal cord syndrome due to abnormal tissue attachments and traction of the cord within the spinal column.

- Symptoms vary including leg or perineal pains, weakness, sensory deficits, sphincter dysfunction or cutaneous stigmata of spinal dysraphism

- Onset may be precipitated during pregnancy by fetal head compression, positioning during child birth or epidural itself
\end{abstract}

\section{CASE DESCRIPTION}

A 33-year-old primip parturient presents for induction of labour at full term in a tertiary women's hospital. Because of a chronic history of neck ache, backache, lower limb pain and weakness, she was extensively worked up to a diagnosis of PMC and hypokalaemia periodic paralysis.

She was also found to have a coexisting history of cervical myelopathy and an incidental spinal cord lipoma extending from filum terminale at $L 2$ to sacrum revealed on MRI in 2010. Otherwise, her other previous investigations were normal including autoimmune serology, cervical muscle electromyography, nerve conduction and transcranial magnetic stimulation studies.

Following an early anaesthetic review to discuss various labour analgesia options and anaesthetic techniques should she require surgery, a plain epidural catheter was inserted between L3 and L4 using loss-of-resistance technique to saline in the sitting position. Hypoaesthesia was confirmed at T10 dermatome. Subsequently, she required an emergency caesarean section for failure to progress. Prior to surgical incision, a satisfactory anaesthesia to T4 dermatome was achieved and an uneventful caesarean section was performed with delivery of a healthy boy.

On post-operative day 1 , she continued to have prolonged bilateral lower limb weakness of medical research council grade 1. Her sensorium and anal tone was intact. An urgent MRI lumbar spine performed ruled out epidural collection or significant spinal canal narrowing. Her lower limb power resolved spontaneously on post-operative day 4 and she was discharged a day after.

\section{DISCUSSION}

There is little published data on obstetric patients with PMC undergoing caesarean section. Grace et al reported an uneventful recovery after spinal anaesthesia for elective caesarean section in a patient with PMC. [3]

Faced with a full-term obstetric patient about to undergo induction of labour, we were limited by time and the lack of definitive investigations. PMC predisposes patient to myotonias exacerbated by exercise and cold. Therefore, we took meticulous care to avoid shivering and ensure adequate warming methods

Lehmann Horn and Lazzo et al reported that there is no increased susceptibility to malignant hyperthermia in patients with PMC. [4] PMC affects mainly the sodium channels, and for that reason is distinct from the risks of malignant hyperthermia due to calcium channel abnormality. Yet, without prior clear classification of her condition, we preferred to err on the side of caution and took special care to avoid any risks of triggering malignant hyperthermia and hyperkalaemia.

Our first choice technique was regional anaesthesia over general anaesthesia. By doing so, we avoided the following medications

1. Use of volatiles especially halothane which commonly produced post op shivering

2. Use of suxamethonium due to its hyperkalaemic effect that can induce myotonias and triggering malignant hyperthermia

3. Use of neostigmine which could potentiate myotonias

Lipoma of the terminal filum is often an incidental finding of no clinical concern. However in some symptomatic patients, it may be associated with tethered cord syndrome in which the conus is often low-lying and more posteriorly displaced within the spinal canal. The general principles are that regional anaesthesia is not contraindicated, however it is important to ensure the following:

1. Document patient's pre-procedure neurological examination carefully

2. Ensure informed consent including a discussion on increased risk of spinal cord trauma

3. An epidural-only technique over a spinal is preferred in order to spare the dura in case of any spinal cord tethering

4. To perform the block below the level of the conus or as low as possible 5. To remove needle or catheter immediately if there is pain on insertion

The genetic defect in PMC also maps to the same gene locus on chromosome 17 as with the other potassium periodic paralyses. Hence strict monitoring of potassium peri-operatively is important.

\section{CONCLUSION}

This is a rare case of an obstetric patient with PMC and a terminal filum lipoma complicated by a spontaneously resolving normokalaemic periodic paralysis.

It is important to adopt an early multidisciplinary approach involving the anaesthetic, obstetric, neurology and neonatology teams to provide the patient with informed decisions and reasonable expectations regarding the delivery and labour. An individual care plan should be formulated by the multidisciplinary team to include plans for timing, mode of delivery and perioperative care.

\section{REFERENCES}

1. Jurkat-Rott K, Lehmann-Horn F. State of the art in hereditary muscle channelopathies. Acta Myol. 2010;29(2):343-50.

2. Adams R D, Victor M, Ropper H. The hereditary myotomas and periodic paralyses. (The channelopathies) In : Principles of Neurology, 6th ed 1997: M C Graw Hill Newyork; 1997:54: 476-488

3. Grace RF, Roach VJ. Caesarean section in a patient with paramyotonia congenita. Anaesth Intensive Care. 1999 Oct;27(5):534-7

4. Lehmann Horn F, Laizzo PA. Are myotonias and periodic paralysis associated with susceptibility to malignant hyperthermia. Br J Anaesth 1990; 65: 692-697

5. Eulenburg A. Über eine familiare durch 6 generations ver-folgbare form congenitaler paramyotonie. Neurologisches Centralblatt 1886; 12:265-272.

6. Thomas A, Leopold U, Winkler H. Malignant hyperthermia in paramyotonia congenita. Anaesthesiol Reanim 1988; 13:295-300.

7. Schieren, Mark; Defosse, Jerome; Böhmer, Andreas; Wappler, Frank; Gerbershagen, Mark U. Less. Anaesthetic management of patients with myopathies. European Journal of Anaesthesiology. 34(10):641-649, October 2017 